\title{
Biologically Inspired Model for Crater Detection
}

\author{
Yang Mu, Wei Ding, Dacheng Tao, and T.F. Stepinski
}

\begin{abstract}
Crater detection from panchromatic images has its unique challenges when comparing to the traditional object detection tasks. Craters are numerous, have large range of sizes and textures, and they continuously merge into image backgrounds. Using traditional feature construction methods to describe craters cannot well embody the diversified characteristics of craters. On the other hand, we are gradually revealing the secret of object recognition in the primate's visual cortex. Biologically inspired features, designed to mimic the human cortex, have achieved great performance on object detection problem. Therefore, it is time to reconsider crater detection by using biologically inspired features. In this paper, we represent crater images by utilizing the $\mathrm{C} 1$ units, which correspond to complex cells in the visual cortex, and pool over the S1 units by using a maximum operation to reserve only the maximum response of each local area of the $S 1$ units. The features generated from the $\mathrm{C} 1$ units have the hallmarks of size invariance and location invariance. We further extract a set of improved Haar features on each $\mathrm{C} 1$ map which contain gradient texture information. We apply this biologically inspired based Haar feature to crater detection. Because the feature construction process requires a set of biologically inspired transformations, these features are embedded in a high dimension space. We apply a subspace learning algorithm to find the intrinsic discriminative subspace for accurate classification. Experiments on Mars impact crater dataset show the superiority of the proposed method.
\end{abstract}

\section{INTRODUCTION}

Impact craters are topographic features on planetary surfaces resulting from impacts of meteoroids. Crater counts are the only available tool for measuring remotely the relative ages of geologic formations on planets [6]. Large craters have been identified manually on Mars and the Moon [8][15]. However, there are abundant millions of identified small sub-kilometer craters calling for an efficient and reliable computing tool for automatic detection.

In the field of object recognition, crater detection is not just another object to be identified. As a special landform formation, crater shapes frequently depart from circles due to millions of years' erosion. Crater rims could be eroded and unidentifiable. Crater sizes may vary by orders of magnitude. These differences indicate that crater detection needs specialized features to represent crater images and different approaches to process them, compared to traditional object detection. Moreover, extracting representative features is more difficult than other object recognition problems. The difficulties are in three aspects: 1) crater sizes are insignificantly different scales, 2) craters are formed varying in morphology and texture, and 3) the shadow and highlight

Y. Mu and W. Ding are with the University of Massachusetts Boston, 100 Morrissey Boulevard Boston, MA 02125. E-Mail: \{yangmu, ding\}@ cs.umb.edu.

D. Tao is with University of Technology, Sydney, NSW 2007, Australia. E-Mail: dacheng.tao@ieee.org.

T. F. Stepinski is with the University of Cincinnati. OH 45221. E-Mail: stepintz@uc.edu. regions of small sub-kilometer craters may be rotated in remotely sensed images according to the different capturing time by satellites. Extracting features invariant to scales and texture are desirable for the task of accurate crater detection.

Improving crater detection performance is closely related with effective feature construction for crater image representations. A good feature should provide exact and succinct description of the subject, keep the discriminative information, and greatly improve the performance of a crater detection classifier. Many features have been developed, including raw pixel values, global features, and local features. Pixel values keep the original image information, but they cannot efficiently reflect the discriminative information and they are too sensitive to position and scale changes. Global features, such as color, texture, and shape, can perform well on most outdoor based object detection cases, but they cannot tolerate changes in orientation and scale, which are critical to describe craters. Local features such as Scale-Invariant Feature Transform [1] cannot perform well when describing a crater because they ignore the spatial information while the texture gradient is an important component to identify craters. Neither of the global features and local features can achieve significant improvement in comparison with raw pixel value on crater detection task. Biologically inspired models, which were originally proposed in 1980s [7], have demonstrated to be effective for general object recognition through improved biologically inspired features [2].

The biologically inspired feature (BIF) set was proposed by Riesenhuber and Poggio (R\&P Model)[3]. It is a set of features obtained from a feed-forward model of the primate visual object recognition pathway, and achieves top level performance in general object recognition and scene classification. The basic motivation for BIF is to mimic the object recognition of humans or primates in cortex. In the object recognition field, Huang et al. proposed an enhanced biologically inspired feature model [4] which greatly improve recognition rate. In the scene recognition field, Song successfully adopted the C1 units for scene classification [5]. $\mathrm{Mu}$ et al. applied biologically inspired features to face recognition and gait recognition tasks [11] [14]. These biologically inspired features cannot be directly used on crater detection, because of the particularity of the crater images. Sub-kilometer crater images often have low resolutions and they are more sensitive to the gradient of textures.

In this paper, we adopt the biologically inspired model to the crater detection problem. After a set of transformations, a crater image is converted to a set of biological maps with the hallmarks of scale and location invariance. Haar features [16] are applied to calculate the texture difference between regions in the biologically inspired transformed maps. The texture gradient information is crucial to identify the craters while the C1 maps omit it. The proposed biologically inspired Haar features (BIF Haar) can combine the advantages of the biological model and the Haar features to keep more 
discriminative information for a crater image.

The biologically inspired featuresare sampled from the low dimension manifold and embedded in a high dimension space from a set of transformation operations. These transformations make the extracted features incorporate abundant discriminative information as well as more redundant and noisy information. Using a subspace learning method is an appropriate way to find the intrinsic low dimensional subspace space and preserve the discriminative information[9] [13]. We propose a subspace learning algorithm and use Discriminative locality alignment (DLA) [9] to find the intrinsic discriminative subspace by optimizing the local compactness and separation.

This paper is organized as follows. Section 2 explains the crater feature extraction process. The discriminative subspace algorithm is discussed inSection 3. Experimental studies are presented in Section 4. Finally, Section 5 concludes the paper.

\section{CRAter FEAtures}

Scale, rotation and location are the three most important aspects to describe a crater image. If we have a feature that is scale, rotation, and location invariance, then all the craters will be very similar to each other using this feature. It is then straightforward to separate crater images from non-crater images. In Figure 1, we show four crater example images on Mars which are normalized to the same size of 48 by 48 pixels. The crater examples are from the Mars dataset used in our previous work [6]. It is straightforward to observe that a shadow region is a key component for crater detection. The images in Figures 1 (a) and 1 (b) have different scale sizesfor shadows. We can get an image very close to Figure 1 (d) from Figure 1 (a) after a $90^{\circ}$ counter clockwise rotation. If we can tolerate the difference in a small range at the top and bottom of the images in Figures 1 (b) and 1 (c), we can regard the shadow parts are about the same despite of orientation. If there is a feature can tolerate the scale, ration and location simultaneously, these four craters will be similar under this feature description.

In next section, we will introduce the biologically inspired model and our proposed BIF Harr features and discuss how BIF Harr features can achieve scale, rotation, and location invariance.

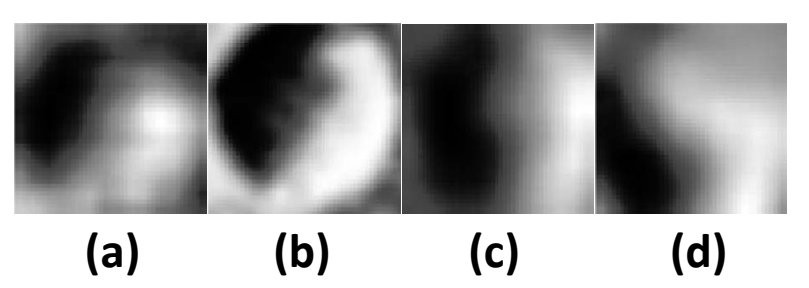

Figure 1. Sample crater images from the Mars impact crater dataset

\section{A. Biologically Inspired Features (BIF)}

As we known, macaques have no problems to recognize objects no matter how the scale, rotation and location change. Experiments show that when macaques recognize objects, the most important part of the brain is the inferotemporal (IT) cortex, the highest purely visual area in the ventral visual stream. The object signal in the cortex is transported to IT through the ventral visual pathway from the primary visual cortex, V1, and extrastriate visual areas V2 and V4. In the area $\mathrm{V} 1$, there are lots of simple cells (S1) and complex cells (C1) [3][7]. The receptive field in the S1 unitsis smaller than that in the $\mathrm{C} 1$ unit. Both cell types respond strongly to oriented bars. Simple cells are with strong position dependence. However, complex cells are invariant to positions and sizes [10].

Specifically, the BIF process is as follows. 1) Using a multidimensional array to represent the input gray value image; 2) obtaining the first layer, the S1 map, by convolving the input multidimensional array with an array of Gabor filters of 4 orientations and 16 scales; 3 ) obtaining the second layer, the $\mathrm{C} 1 \mathrm{map}$, by a maximum operation over the S1 map.

The S1 maps are generated from a convolution operation using Garbor filters. The Gabor function is formally described as:

$$
\begin{gathered}
F(x, y)=\exp \left(-\frac{x_{0}^{2}+\gamma^{2} y_{0}^{2}}{2 \delta^{2}}\right) \times \cos \left(\frac{2 \pi}{\lambda} x_{0}\right) \\
x_{0}=x \cos \theta+y \sin \theta \\
\mathrm{y}_{0}=-x \sin \theta+y \cos \theta
\end{gathered}
$$

where $x$ and $y$ stand for scales; $\theta, \gamma$, and $\delta$ are the orientation, the aspect ratio, and the effective width, respectively; $\lambda$ determines the properties of cells spatial receptive fields.

According to the Garbor function, the S1 map contains different orientations and scales. 4 orientations used in this paperare $0^{\circ}, 45^{\circ}, 90^{\circ}$, and $135^{\circ}$, which contain the information of different angles. 16 scales by varying the range of sizes from $7 \times 7$ to $37 \times 37$ pixels with a step size of 2 pixels which incorporates different scales.

Combining 4 orientations and 16 scales, we can obtain 64 Gabor filters to model 64 receptive fields in the following way:

4 orientations $\times 16$ scales $=64$ Gabor filters.

By convolving the initial input image with the resulting 64 Gabor filters, we generate 64 feature maps in the S1 units with 16 scales and 4 orientations.

After the convolution operations, a large amount of orientation and scale information are incorporated into the S1 map. The S1 map still does not contain the invariance characteristics. The $\mathrm{C} 1$ map corresponding to complex cells in the visual cortex have the tolerance to different scales and locations. We further extract the $\mathrm{C} 1$ map from the S1 maps through a nonlinear MAX operation between adjacent S1 maps which get the max response of the two adjacent $\mathrm{S} 1$ maps scale. This operation make the $\mathrm{C} 1$ feature have the invariance of the proposed characters. An example is illustrated in Figure 2.

We can get the scale invariance with the change of scales by pooling over the $\mathrm{S} 1$ units at the same preferred orientation but responding to different scale of filters. The range of filter sizes determines the degree of invariance provided by the MAX mechanism operations. We combine two adjacent filter scales into a filter band to apply the MAX operation. For 


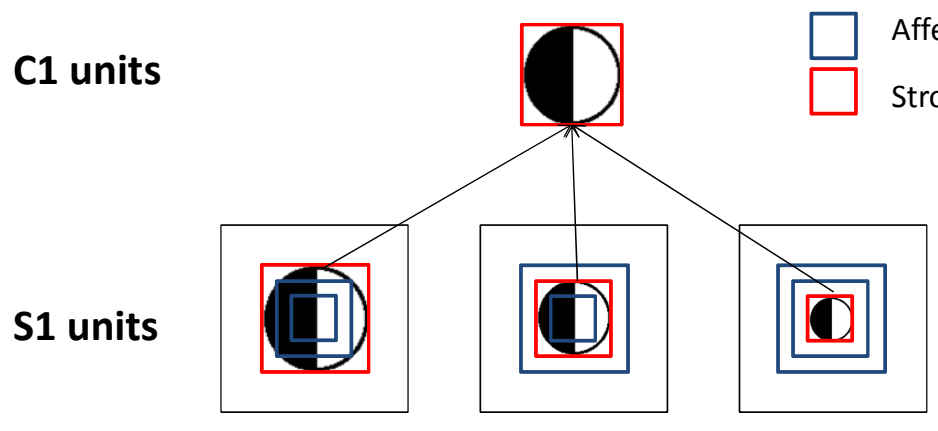

(a)

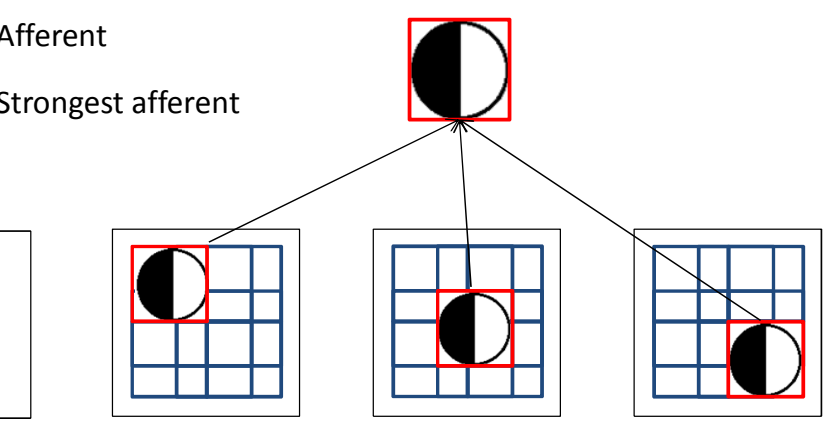

(b)

Figure 2. The process to achieve scale and location invariance at the complex (C1) cells for one crater image: (a) Strongest afferent from different scales fed into the $\mathrm{C} 1$ units to achieve the scales invariance (b) The $\mathrm{C} 1$ units pool over strongest afferent according to different pooling ranges to achieve location invariance

instance, over each orientation, the 16 filters ranging from $7 \times 7$ to $37 \times 37$ are divided into 8 filter bands. The $1^{\text {st }}$ band contains two S1 filters with the size of $7 \times 7$ to $9 \times 9$. The $\mathrm{C} 1$ units can get the size invariant hallmark by pooling on different sizes of filters.

We can get the location invariance with change of location by pooling over the $\mathrm{S} 1$ units at the same preferred orientation but responding to a grid neighborhood location. The grid controls the pooling range. The $\mathrm{S} 1$ units feed into the $\mathrm{C} 1$ units by pooling with the same preferred orientation from all the filters in a filter band. The pooling grids $\mathrm{N}_{S} \times \mathrm{N}_{S}$ span a range of sizes from $8 \times 8$ to $22 \times 22$ in this paper with a step size of 2 pixels. The $\mathrm{C} 1$ map can get the location invariance hallmark by pooling on a pooling grid from the neighborhood location.

In total, we get $32 \mathrm{C} 1$ maps from $64 \mathrm{~S} 1$ maps. From the $\mathrm{C} 1$ map, we can find that the advantages of $\mathrm{C} 1$ map are obvious because they are invariant to the scales and locations of craters.

\section{B. Improved Biologically Inspired Model}

In the Biologically inspired model, the scale and the orientation determine the function of the $\mathrm{S} 1 \mathrm{map}$, and the pooling operation from the $\mathrm{S} 1$ map to the $\mathrm{C} 1$ map is the key component of the model.

To accommodate the 12.5 meters/pixel resolution of Mars crater dataset, we adjust the BIF model and propose two achievements. First, 16 scales of Garbor filters starting from $3 \times 3$ instead of the $7 \times 7$ in the original biologically inspired model which will keep more useful scale information in low resolution crater images. In addition, the MAX pooling grid size $\mathrm{N}_{\mathrm{S}}$ is adjusted to $2 \times 2$ and $4 \times 4$. The size $2 \times 2$ is used for the first 4 filter bands and $4 \times 4$ is applied to the rest 4 filter bands. We choose such a small number to make the biologically inspired model work for relatively low resolution images. In our crater dataset, all the crater images are resized to 48 pixels by 48 pixels. The process to transform the original crater image to the $\mathrm{S} 1$ and $\mathrm{C} 1$ maps is shown in Figure 3.

\section{Haar Basis Features and New Approach}

Ding et al. used Haar basis features for the crater detection task [16]. These features can be thought of as image masks consisting of black and white sectors. 9 types of square masks used are shown in Figure 4.

The difference between black and white sectors will embody the gradient texture which is the best way to reflect the influence of shadow part.

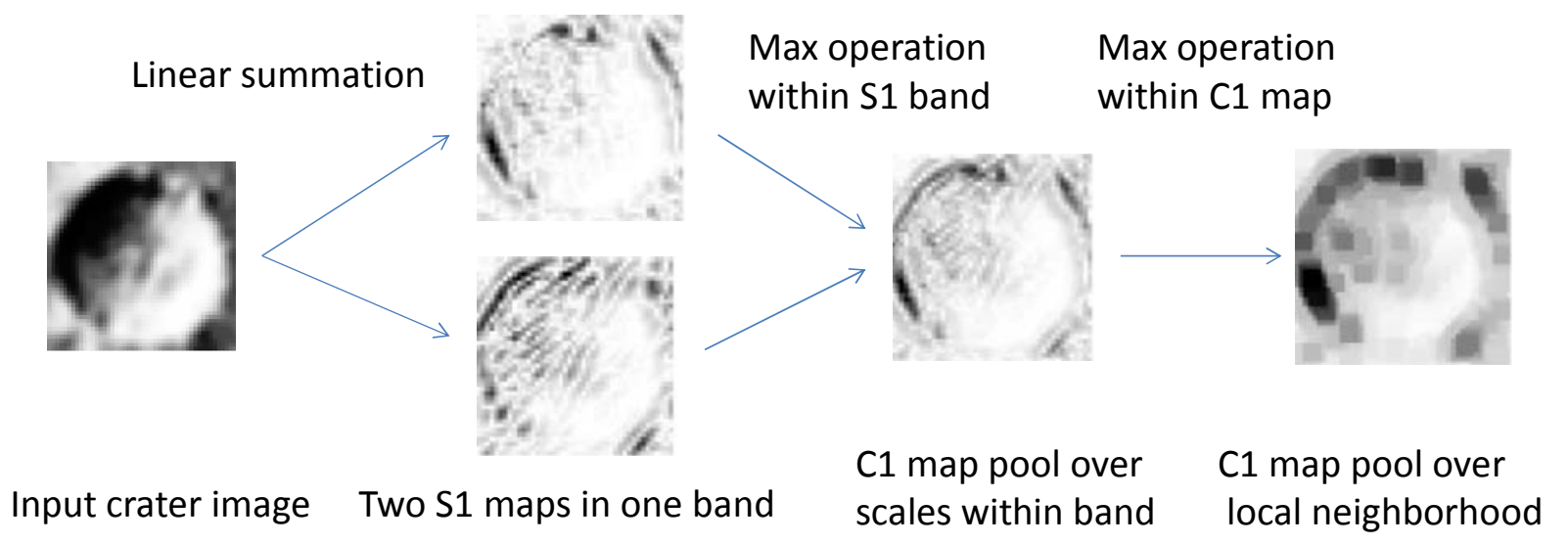

Figure 3. The generation of S1 and $\mathrm{C} 1$ maps for a sample crater image in the $135^{\circ}$ orientation. In one band, two S1 maps are filtered with filter size of $3 \times 3$ and $5 \times 5$. These two S1 maps are fed into the $\mathrm{C} 1$ map by a MAX operation. The $\mathrm{C} 1$ map pool over the local neighborhood to form the final $\mathrm{C} 1$ map. 


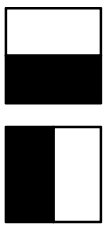

(a)

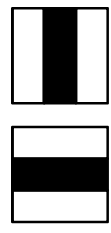

(b)

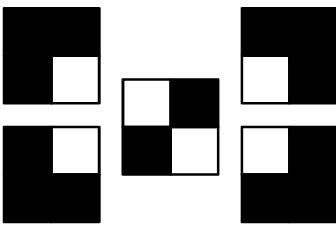

(c)

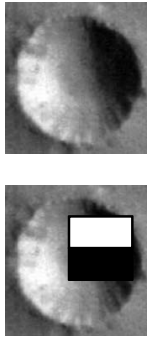

Figure 4. 9 types of square masks: (a) 2 two-rectangle masks to capture horizontal and vertical gradient texture, (b) 2 three-rectangle masks to capture horizontal and vertical edge gradient texture, (c) 5 four-rectangle masks to capture diagonal gradient texture. Far-right: An example of a two-rectangle mask overlay on a crater. A crater is a depression in the surface and appears in the image as a pair of shadow and highlight semi-circular shapes. The illumination is from north-east.

In this paper, we propose a variation for the original Haar mask to make the Haar masks tolerate the rotation variance. From the image mask Figure 4 (a), we can find if the rotated shadow part still falls into the same black or white sector in the image mask, then the difference between black and white sectors will not change. However, if the rotated shadow part falls into the opposite sector, the gradient texture will approximate to

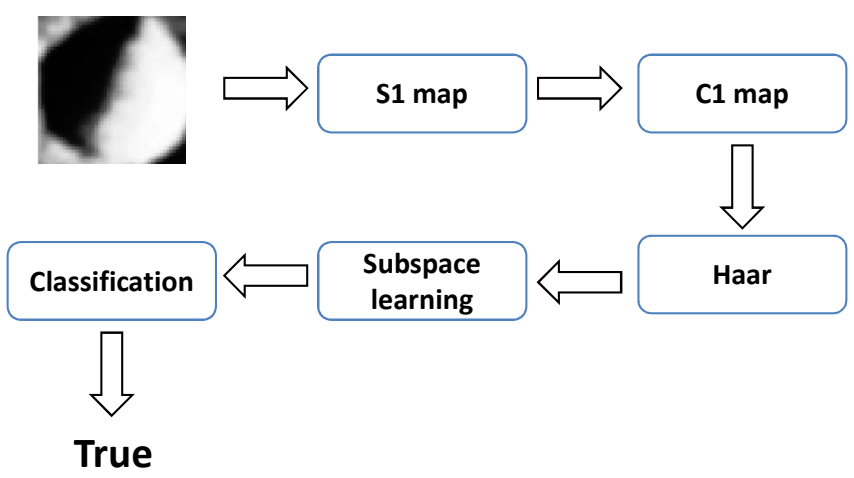

Figure 5. The crater detection framework

the opposite number of previous subtraction, since the shadow part has the dominant pixel intensity. Therefore we propose an improved Haar mask which takes an absolute value of the subtraction to cope with rotation variance.

\section{BIF Haar Features}

The C1 maps can achieve scale and location invariance.In addition, taking an absolute value on Haar basis features can have rotation invariance to some extent. We propose the BIF Haar features which extract Haar features on every C1 map.

Our system for crater classification works in five steps (Figure 5). The input crater image will be firstly converted to $64 \mathrm{~S} 1$ maps and further converted to $32 \mathrm{C} 1$ maps. 1, 089 dimensional absolute Haar features are extracted on each $\mathrm{C} 1$ map. The final set of features to represent a crater candidate image is:

32 map $\times 1,089$ dimension $/$ map $=34,848$ dimensions. We further apply asubspace learning method to identify intrinsic discriminative subspace for this high dimension feature space.

\section{DISCRIMINATIVE SUBSPACE LEARNING}

The dimensionality of the BIF Haar features is rather high and such high dimensional featuresare drawn from low dimension inputs through a battery of transformations. These biologically inspired transformations are imported to enhance the discriminative information. However, much redundant and noisy information is increased simultaneously.

Discriminative information refers to the features that can embody the difference between craters and non-craters. These features will contribute most to a classifier. Redundant features highly increase computational complexity. Noisy features refer to those features that cannot separate the characteristic of craters from non-craters. Noisy features may confuse a classifier thus mislead the prediction results. Redundant and noisy features may reduce the positive impact of the discriminative features and enlarge the distance between true craters. We propose a subspace learning method to find the intrinsic subspace of the BIF Haar features and then project the original features into a low dimension space which incorporates the discriminative information and reduce the redundant and noisy information. In general, linear dimension reduction can be described as follows. Given a set of samples $X=\left[\boldsymbol{x}_{1}, \ldots, \boldsymbol{x}_{n}\right]$, each sample belongs to one of the two classes (crater or non-crater)and is represented as an $m$-dimension vector. Our goal is to find a projection matrix $W$ mapping $X \in \mathbb{R}^{m \times n}$ to $Y \in \mathbb{R}^{d \times n}$, i.e., $Y=W^{T} X$, where $d<m$. The samples in low dimension representation are $Y=\left[\boldsymbol{y}_{1}, \ldots, \boldsymbol{y}_{n}\right]$.

Discriminative locality alignment (DLA) [9] algorithm operates in two steps: First step tries to minimize the distance within class and maximize the distance between classes in a small local patch. Second step tries to generate a final global model by aligning alllocal patches. The objective of this algorithm is to achieve the local compactness and local separation in a discriminative subspace. This subspace is expected to keep the discriminative information and reduce the noisy and redundant information.

\section{A. Local Optimization}

For a given training sample $\boldsymbol{x}_{i}$, according to the label information of $\boldsymbol{x}_{i}$, we can build a local patch $X_{i}$ which incorporates $k_{1}$ same class nearest neighbors of $\boldsymbol{x}_{i}$, and $k_{2}$ different class nearest neighbors of $\boldsymbol{x}_{i} . X_{i}=\left[\boldsymbol{x}_{i}, \boldsymbol{x}_{i^{1}} \ldots \boldsymbol{x}_{i^{k_{1}}}, \boldsymbol{x}_{i_{1}} \ldots \boldsymbol{x}_{i_{k_{2}}}\right]$ and $X_{i} \in \mathbb{R}^{m \times\left(1+k_{1}+k_{2}\right)}$. In this local patch, the local compactness is reflected by the distance between the given sample $\boldsymbol{x}_{i}$ and its $k_{1}$ nearest neighbors in identical class. The local separation is reflected by the distance between the given sample $\boldsymbol{x}_{i}$ and its $k_{2}$ nearest neighbors in a different class. Therefore, the local scatter matrix for local patch $X_{i}$ is defined as

$$
S_{L_{i}}=\sum_{p=1}^{k_{1}}\left(\boldsymbol{x}_{i}-\boldsymbol{x}_{i} p\right)\left(\boldsymbol{x}_{i}-\boldsymbol{x}_{i^{p}}\right)^{T}-\beta \sum_{p=1}^{k_{2}}\left(\boldsymbol{x}_{i}-\boldsymbol{x}_{i_{q}}\right)\left(\boldsymbol{x}_{i}-\boldsymbol{x}_{i_{q}}\right)^{T},
$$


where $\beta$ is a scaling factor to unify the different measure of local compactness and local separation. Let the coefficients vector $\omega=[\overbrace{1, \cdots, 1}^{k_{1}}, \overbrace{-\beta, \cdots,-\beta}^{k_{2}}]^{T}$, then we have
$\begin{gathered}S_{L_{i}}=\sum_{j=2}^{k_{1}+k_{2}+1} \omega\left(X_{i}\{1\}-X_{i}\{j\}\right)\left(X_{i}\{1\}-X_{i}\{j\}\right)^{T} \\ =\sum_{j=2}^{k_{1}+k_{2}+1} X_{i} L_{i} X_{i}{ }^{T}\end{gathered}$

where $X_{i}\{j\}$ is the $j^{\text {th }}$ element of $X_{i}, L_{i}$ is given by

$$
\left[\begin{array}{cc}
\sum_{j=1}^{k_{1}+k_{2}+1} \omega_{j} & -\omega^{T} \\
-\omega^{T} & \operatorname{diag}(\omega)
\end{array}\right] .
$$

\section{B. Global optimization}

When aligning all the local scatter matrices, we can get the final local scatter matrix

$$
S=\sum_{i} S_{L_{i}}
$$

After applying the projection by matrix $W$, the local scatter of the transformed feature $Y$ is $W^{T} S W$.

The projection matrix $W$ is chosen to minimize the trace of the global scatter matrix as well as the local scatter matrix.

To make the projection matrix $W$ linear and orthogonal, we can impose the term $W^{T} W=I_{m}$, where $I_{d}$ is a $m \times m$ identity matrix. The final objective function is given by

$$
\arg \min _{W} \operatorname{tr}\left(W^{T} S W\right) \text { s.t. } W^{T} W=I_{m}
$$

Optimal solutions are given by using the standard eigen-decomposition:

$$
S \boldsymbol{u}=\lambda \boldsymbol{u} .
$$

Let the column vectors $\boldsymbol{u}_{1}, \ldots, \boldsymbol{u}_{m}$ be the solution ordered according to eigenvalues $\lambda_{1}<\lambda_{2}<\cdots \lambda_{m}$. The optimal projection matrix $W$ is then given by $W=\left[\boldsymbol{u}_{1}, \ldots, \boldsymbol{u}_{m}\right]$. This projection matrix can project the data into a discriminative subspace which will greatly help the classification.

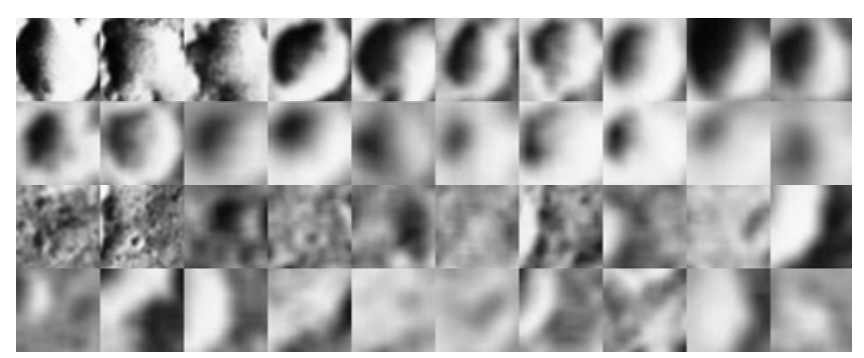

Figure 6.Sample crater images. The top two rows correspond to real craters image. The bottom two rows correspond to non-craters images

\section{EXPERIMENTS}

To test the performance of the new biologically inspired model, we used a portion of the HRSC nadir panchromatic, $12.5 \mathrm{~m} /$ pixel image of Mars (\# h0905_0000) [17]. In this scene we have manually cataloged 3,050 craters having diameters between 40 to 6,600 meters. The Nanedi Valles passes through the middle of the scene introducing some heterogeneity of the terrain. In order to account for this heterogeneity we have divided the scene into three sections labeled West, Central (containing Nanedi Valles), and East. West range and East regions are similar in morphology and texture, while West range has more smallcraters. Central range contains a valley which make the craters varies a lot in shapes and textures.

Totally 2,085 true craters are extracted as positive samples in this crater dataset. Most of these craters are sub-kilometer craters and are chosen from the west region. Non-craters are selected according to dense sample the crater images. The 2,085 negative samples which may partially overlap with the true craters are extracted to build the negative samples for this crater dataset ${ }^{1}$. The ground truth crater can be found in Figure 7. A pre-processing operation is further applied to crop the circular range of the image to emphasize the shadow part inside the craters. We adopt 10 -fold cross-validation to do the comparative experimental study. The number for training samples varies from $10 \%$ to $90 \%$ of the size of the whole dataset.

Experimental results are demonstrated in three aspects. 1) the feature comparison among biologically inspired features, Haar features and pixel based features; 2) Our proposed new BIF Haar model versus the original biologically inspired features model; and 3) the experimental results comparison between the proposed biological inspired model and the Haar-boosting pipeline [6].

The performance of biologically inspired features, Haar features and pixel based features are compared in this section.The discriminative subspace learning algorithm DLA is compared with the Boosting algorithm which is the best algorithm so far on the benchmark dataset [17] for crater detection [6]. The classifiers in used for our proposed method is the $\mathrm{K}$-nearest neighbor $(\mathrm{KNN})$ classifier. Totally the BIF Haar features have 34,848 dimensions which are extracted

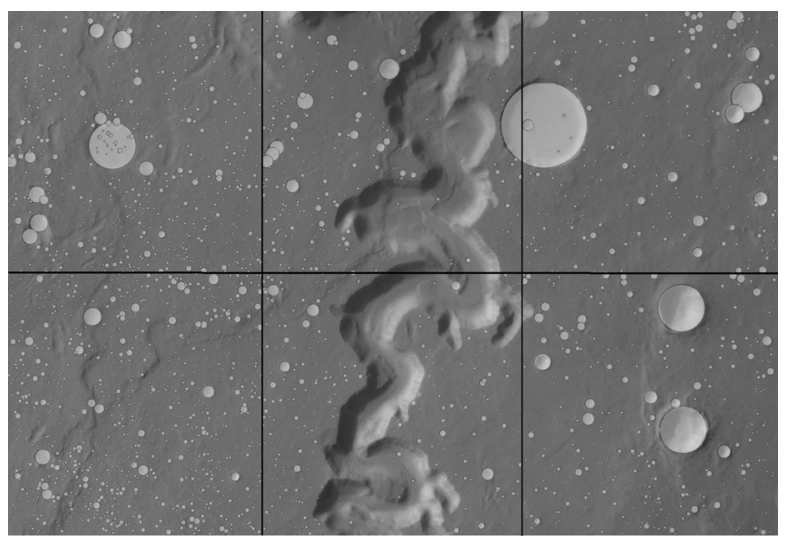

Figure 7. The ground truth figure of Mars impact crater dataset

based on $32 \mathrm{C} 1$ maps and 1,089 Haar features on each $\mathrm{C} 1$ map.

\footnotetext{
${ }^{1}$ http://www.cs.umb.edu/ yangmu/dataset/
} 


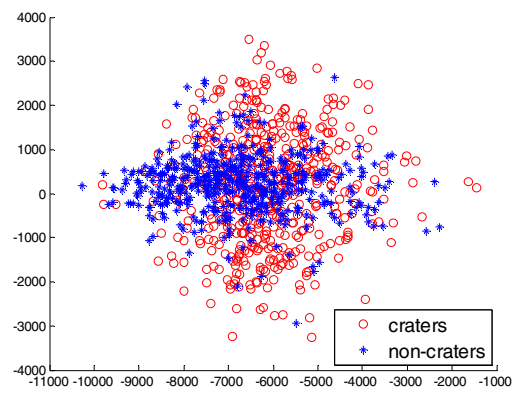

(a)

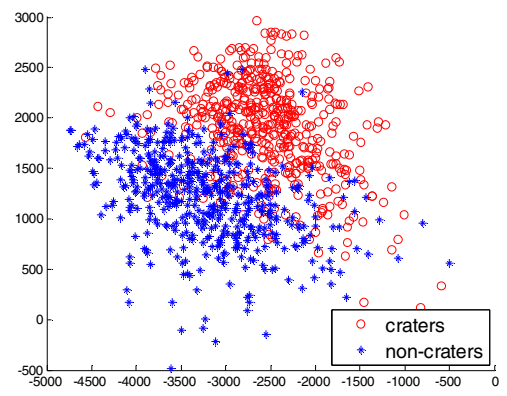

(b)

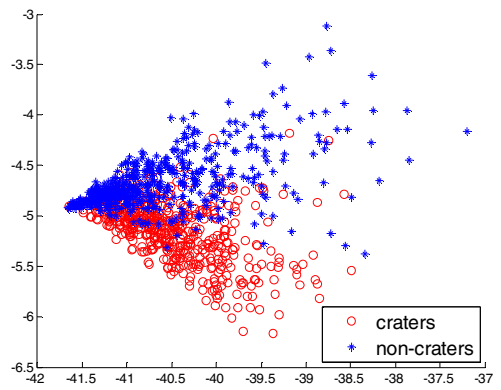

(c)

Figure 8.Impact dataset visualization under different features in a 2-dimension PCA space. (a). Using pixel directly to describe the crater images. (b). Extracting Haar feature to describe craters images. (c). Extracting Biologically inspired features to describe crater images.

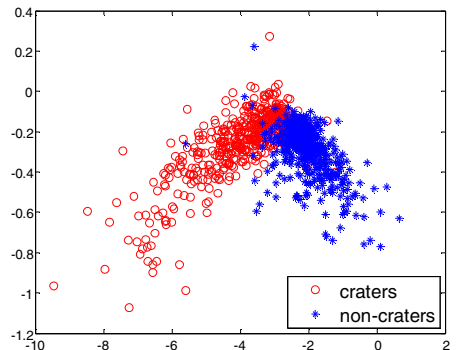

(a)

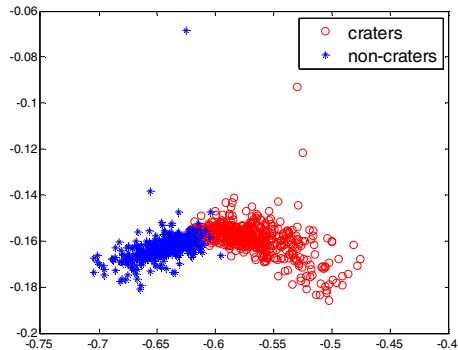

(b)

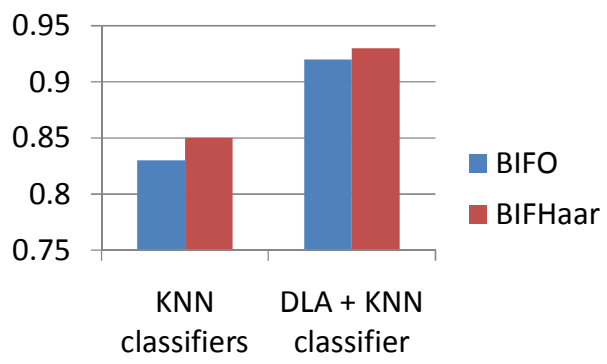

(c)

Figure 9. Impact dataset visualization under different biological inspired features on 2-dimension DLA space. (a). The visualization of the original biologically inspired features. (b). The visualization of proposed features combining biological inspired patterns and Haar texture information (BIF Haar). (c). Comparison results between the original biological inspired model and our proposed BIF Harr model.

\section{A. Feature Space Distribution Visualization}

The biologically inspired features are features to mimic theway human beings recognize object. Human beings have no difficulty to identify a crater image no matter how the scale, location and rotation angle change. These biologically inspired features through a set of transformations should keep this characteristic. However, the raw pixel features and Haar features lack of such invariance hallmarks. To justify our assumption, we project all the samples in the crater dataset to these three features spaces and analyze the visualization of distribution.

The visualization results are shown in Figure 8. In the visualization process, all the crater images are projected into a two-dimension Principal Component Analysis (PCA) space. In the two-dimension PCA space, two orthogonal directions corresponding to the largest variance for all the samples are selected as the coordinates. This visualization can use less dimensions to keep the most principal components which can best represent the original feature distribution.

From Figure 8, we can find that Haar features and the biologically inspired features can clearly make the true craters and non-craters have different distributions. While pixel based features fail to clearly reflect the different distributions of true craters and non-craters. Furthermore, the biologically inspired features make the data more aggregated and have smaller overlapping margin than the Haar features. Since PCA does not take the label information into consideration, the distribution visualization cannot fully exploit the discriminative information. Under the PCA space, when the number of training sample is $90 \%$ of the whole dataset, the accuracy of the biologically inspired features is 0.86 , the Haar feature's accuracy is 0.87 , while the pixel based feature is 0.84 . The biologically inspired features method is comparable with the Haar features and both of them can capture the two different distributions of true craters and non-craters. If label information is taken into consideration, more discriminative information could be utilized to identify these two distributions, which is discussed in part $\mathrm{C}$ of this section.

\section{B. Biologically Inspired Model Comparison}

There are two biologically inspired models introduced in this paper: the original biologically inspired model (BIFO), our proposed model with combination of advance pooling range, and the Haar texture information (BIF Haar).

We compare these two biologically inspired features by the proposed discriminative subspace method and their original features space. The principle components of both the biologically inspired approaches are similar and theyhave the similar PCA projection as shown in Figure 8(c). When considering the label information, different classification abilities can be exploited. The projection on the 


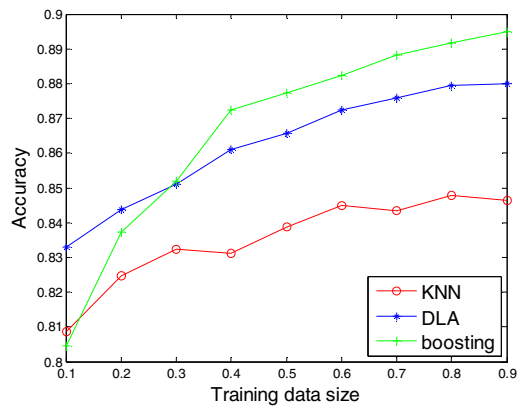

(a)

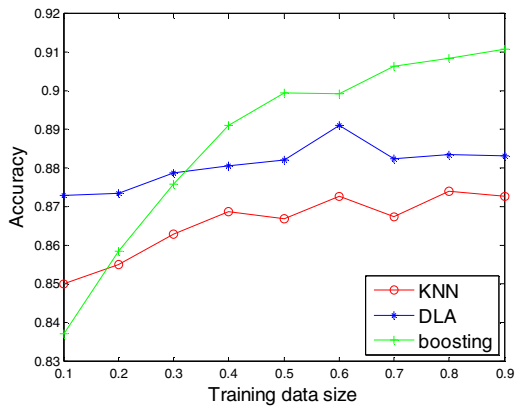

(b)

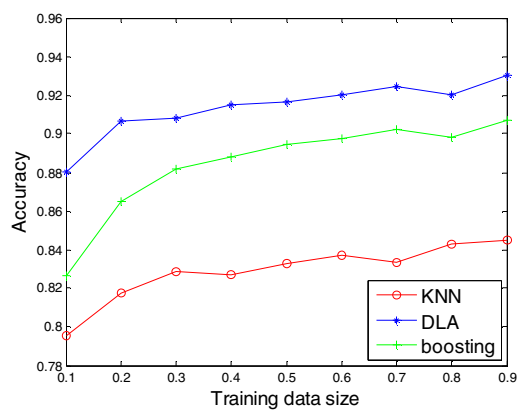

(c)

Figure 10. Impact crater dataset classification accuracy versus the size of the training set. (a) The comparison results of the three algorithms with the pixel based features. (b) The comparison results of the three algorithms with the Haar features. (c) The comparison results of the three algorithms with the BIF Haar features.

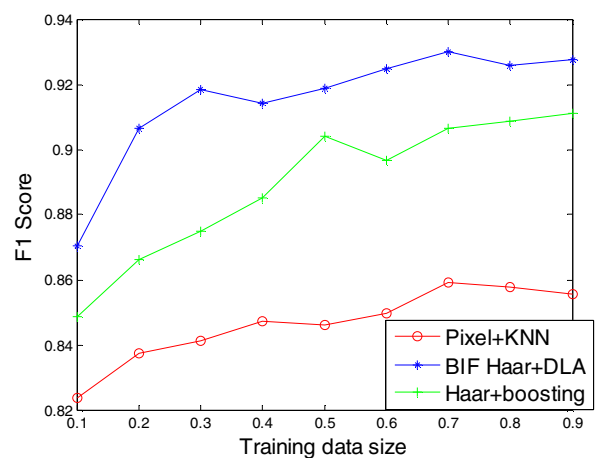

(a)

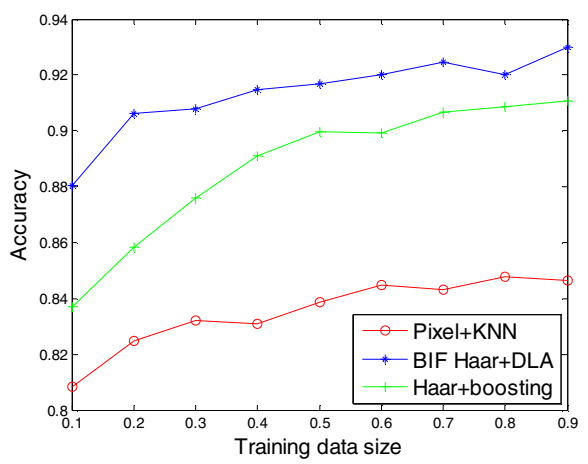

(b)

Figure 11. F1 Score and accuracy comparative results versus the size of training data. Pixel $+K N N$ method is the baseline method. (a) The F1 score of these three methods. (b) The classification accuracy of these three methods.

discriminative subspace is visualized in Figure 9.

From the generation of biologically inspired based approaches, we know these features would contain more discriminative information based on a set of transformations. The DLA algorithm can exploit the discriminative subspace. From the original BIF and the BIF Haar DLA feature space projection shown in Figures 9 (a) and 9 (c), the intrinsic discriminative space can be found to clearly separate the two different distributions of craters and non-craters. BIF Haar features in Figure 9 (b) has more compactness than the BIFO features which means the overlap between craters and non-craters is reduced and samples from either craters or non-craters are more easier to be identified.

Experiments are designed to evaluate BIF Haar and BIFO. Training samples are $90 \%$ of the total dataset. We apply the KNN classifier directly upon the features to test the performance of these features. Furthermore, we apply the KNN classifier on the projected DLA subspace for both features to examine the ability of incorporating discriminative information. Experimental results are shown in Figure 9 (c). Haar texture features can complement the disadvantage of the original biologically inspired features by adding the texture information and rotation invariance hallmarks. Therefore, the proposed BIF Haar features are superior to the original BIF both on the original feature space and the projected discriminative subspace.

\section{Crater Detection FrameworkComparison}

One of the best existing crater detection frameworks is reported in [6], which adopts the Haar features to describe the crater images and utilize boosting algorithm to classify the crater candidates. In the experiments in this paper, the number of training samples varies from $10 \%$ to $90 \%$ of the total crater dataset. Craters and non-craters samples are selected in a random and balance scheme. There are two algorithms involved with this comparison: discriminative subspace based DLA algorithm versus boosting method. The Euclidean distance plus KNN classifier method is used as a baseline method. Local patch size parameters used in the DLA algorithm are $\mathrm{k} 1=7$ and $\mathrm{k} 2=2$. The parameters of boosting algorithm are the same as the original paper. The experimental results could be found in Figure 10.

Figure 10 (a) tests the performance of different algorithms on the original pixel feature space. In this space, there are a lot of redundant and noisy information which will influence the performance of algorithms. We can find that the subspace learning method and the boosting method are comparable under this feature space. Boosting method will perform much better when the training samples are sufficient while when the training samples are limited boosting method will fail to generate a strong classier. Since boosting method uses an ensemble way to combine the weak learners to produce a better prediction. If the training samples are limited, the learned weak learners may not represent the whole dataset 
which will lead to the over-fitting problem.

The performance of these three methods on Haar features is shown in Figure 10 (b). The Boosting method achieves the best overall results on Haar features. This result is consistent with [6]. The Haar features calculate the difference between two regions. If one of the two regions contains the shadow part of the crater, then this Haar feature will reflect much discriminative information and a weak learner with good performance can then be derived from this feature. Therefore, boosting method will achieve the best results for the Haar features.

Figure 10 (c) illustrates the performance for these methods on the BIF Haar features. Since the BIF Haar features have a very high dimension space, abundant discriminative information and redundant are incorporated. DLA can find a discriminative subspace to reduce the redundant information while keeping the discriminative information which best suit this feature. Therefore, DLA achieve best result for BIF Haar features.

We compare the Haar+boosting method with our proposed BIF Haar+DLA method in Figure 11. Figures11 (a) and 11 (b) give the results of the F1 score and accuracy of the three methods versus the size of training data. F1 score is the harmonic mean of the precision and recall. F1 score and accuracy are both very important statistical ways for analyzing crater detection performance. The F1 score reflect the performance of the classifier to deal with the positive crater samples. The accuracy measures the prediction ability. For a classifier report a TP has greater impact to report a TN. The formulas of the evaluation metrics are as follows.

$$
\begin{aligned}
& \text { Precision, } p=\frac{\mathrm{TP}}{\mathrm{TP}+\mathrm{FP}} \\
& \text { Recall, } r=\frac{\mathrm{TP}}{\mathrm{TP}+\mathrm{FN}} \\
& \mathrm{F} 1=2 \frac{p \times r}{p+r}=\frac{2 \mathrm{TP}}{2 \mathrm{TP}+\mathrm{FP}+\mathrm{FN}} \\
& \text { Accuracy }=\frac{\mathrm{TP}+\mathrm{TN}}{\mathrm{TP}+\mathrm{FP}+\mathrm{FN}+\mathrm{TN}}
\end{aligned}
$$

A test crater candidate is counted as a True Positive (TP) if it is a true crater and the classifier correctly labels this candidate as a positive. False Positive (FP) refers to a non-crater candidate which is misclassified as a crater. True Negative (TN) means the non-crater candidate which is also correctly predicted as a non-crater by classifier. False Negative (FN) is a true crater candidate which is predicted as a non-crater by classifier.

We can find that our proposed method greatly outperforms the Haar+boosting method in both F1 score and accuracy in Figure 11.The experimental results justify the proposed biologically inspired features and the DLA learning algorithm can achieve better results than the existing method.

\section{CONCLUSION}

In this paper, we have presented a new framework for automatic crater detection constructed from biologically inspired Haar features using the DLA algorithm. For the feature selection part, we applied the $\mathrm{Cl}$ units, which correspond to the complex cells in the visual cortex and have the good hallmarks of invariance to the change of positions and sizes. We further extract the Haar features on each $\mathrm{C} 1$ map which make the feature invariant to the rotation to some extent. The challenges of crater detection are the great variation of sizes and textures. The $\mathrm{C} 1$ feature plus Haar features have good tolerance to these changes. Since the dimension for these BIF Haar features are extremely high, we apply a discriminative subspace algorithm DLA to find the intrinsic subspace to improve the recognition performance. Experiments ona Mars crater dataset has demonstrated that the new biologically inspired model, works well in the crater detection task.

\section{ACKNOWLEDGMENT}

This work is partially supported byNASA under grant NNX09AK86G.

\section{REFERENCES}

[1] D. Lowe (2003). Distinctive image features from scale-invariant keypoints. International Journal of Computer Vision, 60(2), 91- 10.

[2] T.Serre \& M.Riesenhuber (2004) Realistic modeling of simple and complex cell tuning in the HMAX model, and implications for invariant object recognition in cortex. AI Memo 2004-017/CBCL Memo239,Massachusetts Institute of Technology.

[3] M. Riesenhuber\& T.Poggio, (2000) Models of object recognition. Nature Neuroscience, 3, 1199 - 1204.

[4] Y.Huang, K. Huang, D. Tao, T. Tan\& X. Li (2008) Enhanced biologically inspired model. International Conference on Computer Vision and Pattern Recognition, 1-8

[5] D. Song, \&D. Tao (2009) Biologically inspired feature manifold for scene classification. IEEE Transactions on Image Processing Volume 19 Issue 1, January 2010.

[6] W. Ding, T. Stepinski, Y. Mu, L. Bandeira, R. Vilalta, Y. Wu, Z. Lu, T. Cao, X. Wu, Sub-Kilometer Crater Discovery with Boosting and Transfer Learning.ACM Transactions on Intelligent Systems and Technology, accepted and to appear, 2011

[7] K. Fukushima (1980). Neocognitron: a self organizing neural network model for a mechanism for pattern recognition unaffected by shift in position. Biological Cybernetics, 36(4), 193-202.

[8] Barlow, N. G. 1988. Crater size-frequency distributions and a revised Martian relative chronology. Icarus 75, 285-305.

[9] T. Zhang, D. Tao, X. Li, J. Yang, Patch alignment for dimensionality reduc- tion. IEEE Transactions on Knowledge and Data Engineering 21 (9) (2009) 1299-1313.

[10] R. Schneider,M. Riesenhuber, Detailed look at scale and translation invariance in a hierarchical neural model of visual object recognition. AI Memo 2002-011/CBCL Memo218, Massachusetts Institute of Technology, 2002.

[11] Y. Mu \& D. Tao: Biologically inspired feature manifold for gait recognition. Neurocomputing 73(4-6): 895-902 (2010)

[12] Riesenhuber M, Poggio T. Hierarchical models of object recognitionin cortex. Nature. 1999;2(11):1019-25.

[13] T. Zhang, D. Tao, X. Li, J. Yang. A unifying framework for spectralanalysis based dimensionality reduction. IEEE international joint conference on neural networks; 2008. p. 1671-8.

[14] Y. Mu, D. Tao, X. Li\& F. Murtagh: Biologically Inspired Tensor Features. Cognitive Computation 1(4): 327-341 (2009)

[15] Andersson, L. E. and Whitaker, E. A. 1982. Nasa catalog of Lunar nomenclature.NASA Reference Publication 1097.

[16] Viola, P. and Jones, M. J. 2004. Robust real-time face detection. International Journal of Computer Vision 57, 137-154.

[17] Mars Impact crater dataset. http://hrscview.fu-berlin.de/. 